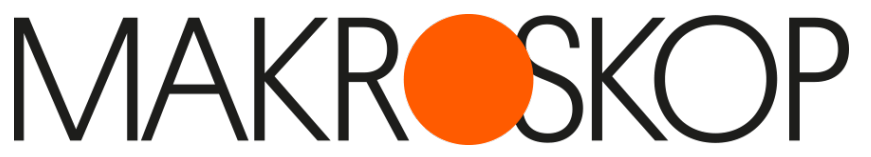

Kritische Analysen zu

Politik und Wirtschaft.

Herausgeber: Heiner Flassbeck

\& Paul Steinhardt

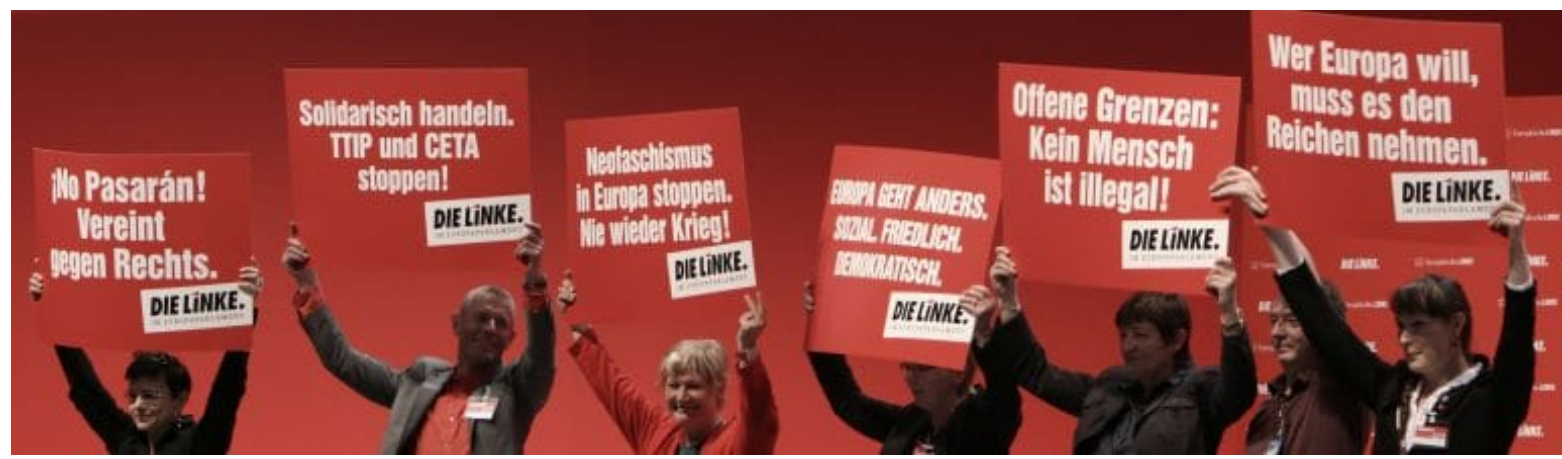

\title{
Welches Europa wollen wir?
}

Von Martin Höpner und Ralf Krämer | 16.01.2019

Der Entwurf des Europaprogramms der LINKEN verdeutlicht, dass es nicht genügt, die EU mit sozialeren Inhalten füllen zu wollen. Vielmehr muss ihre Architektur selbst auf den Prüfstand. Wie sind die in dem Programm dazu gemachten Reformvorschläge zu bewerten?

Auf ihrem Bonner Parteitag vom 22. bis 24. Februar wird die Partei DIE LINKE ein Europaprogramm verabschieden. Das ist über die Partei hinaus für alle von Interesse, die nach progressiven Antworten auf die Krise der Europäischen Union suchen. Daher haben wir uns den hier ab Seite 45 einsehbaren, mit „Für ein solidarisches Europa der Millionen, gegen eine Europäische Union der Millionäre“ überschriebenen Leitantrag des Parteivorstands einmal genauer angeschaut. Wie wir meinen, lassen sich einige Fortschritte, aber auch Probleme der progressiven Europadebatte anhand des Textes gut aufzeigen.

Beginnen möchten wir mit Lob und Anerkennung für die pragmatische Ausrichtung des Entwurfs. DIE LINKE vermeidet es, in die Falle der SPD zu tappen, die ihre Wahlkampagne unter das verheerende Motto „Europa ist die Antwort” (hier) gestellt hat. Frei übersetzt: Egal wie die Fragen lauten - und egal, was wir Sozialdemokraten sonst so erzählen, wenn nicht gerade Europawahlen anstehen -, in diesem Wahlkampf werden wir alles mit „mehr Europa” beantworten.

Der Programmentwurf für den Bonner Parteitag wählt aus guten Gründen einen anderen, besseren Weg: Die europäische Integration soll an der Einhaltung ihres sozialen Versprechens gemessen werden. Es geht um inhaltliche Anforderungen, nicht um Festlegungen auf "mehr" oder "weniger" Euro- 
pa. Wer ein Plädoyer für die „Vereinigten Staaten von Europa“ will, wird in den vorderen, grundsätzlichen Teilen des vorgeschlagenen Programms vergeblich suchen.

Der Entwurf verdeutlicht, dass es nicht genügt, die bestehende Architektur der EU mit sozialeren Inhalten füllen zu wollen. Vielmehr muss ihre Architektur selbst - das sind die Vertragsgrundlagen auf den Prüfstand. Viele Programmpunkte stehen im Einklang mit den Forderungen anderer Parteien und Organisationen des progressiven Spektrums. Bei anderen hat die Partei DIE LINKE zumindest im deutschen Parteienspektrum ein Alleinstellungsmerkmal. Beispielhaft zu nennen wären die Forderungen nach der Einführung eines expliziten Beschäftigungsziels; nach Abschaffung des Fiskalpakts und des Stabilitäts- und Wachstumspakts (man sollte präzisionshalber ergänzen: der sanktionsbewehrten makroökonomischen Korrekturverfahren); und die Forderung nach Ermöglichung direkter Staatsfinanzierung durch die Europäische Zentralbank (EZB) für ausgewählte, präzise zu benennende und eng zu definierende Projekte.

\section{Was soll auf europäischer Ebene geregelt werden?}

Damit haben die Autorinnen und Autoren des Antrags unseres Erachtens einige stimmige Grundentscheidungen getroffen. Werden diese Entscheidungen im weiteren Verlauf des Antragstextes stimmig durchgehalten?

Dazu wäre es notwendig, Punkt für Punkt zu entscheiden, auf welcher Ebene des europäischen Mehrebenensystems Entscheidungen so anzusiedeln wären, dass am Ende möglichst soziale Ergebnisse stehen. In weiten Teilen verfährt das Programm allerdings anders: durch schlichte Projektion der typischerweise an die mitgliedstaatliche Ebene gerichteten Forderungen auf die Europäische Union. ÖPNV, Aufwertung von Erziehungsberufen, Zukunft des Transsexuellengesetzes, Rente, Wohnen, Kohleausstieg, Befristung von Arbeitsverhältnissen, Teilzeit, Leiharbeit, Arbeitszeitverkürzung, Maßnahmen gegen Pflegenotstand, sozialer Wohnungsbau, Massentierhaltung - zu diesen und noch viel mehr Punkten richtet das Programm Forderungen an die europäische Ebene.

Ihrem Inhalt nach mögen all diese Forderungen in Ordnung gehen. Aber wer wollte das alles wirklich auf europäischer Ebene geregelt wissen? Wollen wir, wenn wir im nationalen Rahmen eine Mehrheit für unsere Forderungen erstritten haben, wirklich warten, bis dieselben Mehrheiten in anderen EU-Mitgliedsländern vorliegen, um dann eine europäische Richtlinie verabschieden zu können, die dann national umzusetzen ist?

Zudem: Nehmen wir einmal im Gedankenexperiment an, all das, was im Entwurf von der EU eingefordert wird, würde plötzlich wirklich auf supranationaler Ebene geregelt - mit dem Inhalt allerdings, dass unsere progressiven Lösungen durch die entsprechenden Richtlinien und Verordnungen unterbunden würden (angesichts der Kräfteverhältnisse und des Stands des Primärrechts die realistischere Option!). Würden wir die europäisch bewirkte Einengung unserer Handlungsspielräume gleichwohl als legitim erkennen und verteidigen? Wäre bei der Projektion progressiver Forderungen auf die europäische Ebene daher nicht wesentlich mehr Vorsicht geboten?

Diesem Überschuss an europäischer Gerichtetheit von Forderungen steht unseres Erachtens derzeit noch eine recht hohe Zahl an spezifisch europäischen Entwicklungen gegenüber, über die sich der Pro- 
grammentwurf ausschweigt. Beispielhaft wären zu nennen: Die Vorbereitung neuer Erweiterungsrunden; die Zukunft des Europäischen Stabilitätsmechanismus (ESM) und der Architektur der Eurozone allgemein; Ausmaß und Dauerhaftigkeit der innereuropäischen realen Wechselkursverzerrungen; die Befugnisse und die Autonomie der EZB; die Banken- und die Kapitalmarktunion; die Möglichkeit eines Euro-Austritts Italiens und weiterer südeuropäischer Länder sowie die von der Kommission angestrebte Erweiterung der Eurozone; die Überinterpretation der Binnenmarktfreiheiten und ihre normative Vorrangstellung gegenüber sozialen Grundrechten, dem europäischen Sekundärrecht und dem mitgliedstaatlichen Recht (auf letzteres kommen wir unten noch einmal zurück).

\section{Der falsche Ruf nach einer europäischen Verfassung}

Auch an einer anderen Stelle durchbricht der Programmentwurf den Verzicht auf eine Festlegung auf "mehr" oder "weniger" Europa. Denn spät und gut versteckt im Programmtext, in den Zeilen 1799-1809, will der Entwurf eine „Verfassung für Europa”. Wir gehen davon aus, dass hier lediglich bessere Verträge gemeint sind. Der Ruf nach einer Verfassung ist aber eigentlich etwas anderes und könnte als Plädoyer für die „Vereinigten Staaten von Europa” durch die Hintertür gelesen werden. Staaten haben Verfassungen, anderweitige internationale Konstruktionen Verträge; Staaten haben zudem eine Kompetenz-Kompetenz.

Warum warnen wir davor, diesen Schritt - zumal unter den derzeitigen Kräfteverhältnissen! - zu gehen? Im Prinzip ließen sich die Verträge (der Vertrag über die Europäische Union und der Vertrag über die Arbeitsweise der EU) als europäische Verfassung deklarieren. An der Heterogenität der Arbeitsund Sozialverfassungen der EU-Mitgliedstaaten und damit auch an den begrenzten Chancen einer schrittweisen Vereinheitlichung des Sozialen in der EU würde diese Deklaration allerdings nichts ändern. Vor dem (im Entwurf allenfalls am Rande vorkommenden) Europäischen Gerichtshof (EuGH) würden hingegen alle Dämme brechen, wenn es darum geht, mitgliedstaatliche Rechtsbestände an den Binnenmarktfreiheiten und dem EU-Wettbewerbsrecht zu messen. Am Ende stünde kein Vorteil für die sozialpolitische Aktivierung des europäischen Gesetzgebers, sondern eine erhebliche Beschleunigung der liberalisierenden „Integration durch Recht". Die Forderung nach einer europäischen Verfassung sollten progressive Kräfte daher der Europa-Union und „Pulse of Europe“ überlassen und sich darauf konzentrieren, das soziale Potenzial der EU zu maximieren.

\section{Die Wucht der Binnenmarktfreiheiten eindämmen}

Dass dem Entwurf ein Bezug auf die zentrale Forderung des Europäischen Gewerkschaftsbunds (EGB) nach einem Sozialen Fortschrittsprotokoll fehlt, ist gewiss ein unabsichtliches Versäumnis. Diese Forderung war eine Antwort auf die EuGH-Rechtsprechung in den Fällen Viking und Laval.[1] Seither hat sich die Debatte über die vom EuGH vorgenommene faktische Unterordnung des Streikrechts unter die Binnenmarktfreiheiten weiterentwickelt und die Gegenforderungen wurden auf europarechtlich solidere Grundlagen gestellt.

Die im Entwurf für ein Soziales Fortschrittsprotokoll artikulierte Forderung des Europäischen Gewerkschaftsbunds, das Soziale effektiver vor der Wucht der Wirtschaftsintegration zu schützen, sollte unseres Erachtens von progressiven Kräften und so auch im Europawahlprogramm der Partei DIE 
LINKE offensiv unterstützt werden. Obwohl es uns in diesem Kommentar eher um die groben Linien geht, möchten wir hier eine konkrete Änderung vorschlagen.

Wir regen an, die ab Zeile 730 beginnenden Forderungen in Abschnitt 4, „Menschen vor Profite“, um die Forderung nach so genannten Bereichsausnahmen zu ergänzen, und zwar in folgender Formulierung: Um die Arbeits- und Sozialverfassungen der Mitgliedstaaten vor dem Liberalisierungsdruck der EU-Wirtschaftsintegration zu schützen, müssen sie mittels Vertragsänderungen vom Anwendungsbereich (1) der Binnenmarktfreiheiten, (2) des europäischen Wettbewerbsrechts und (3) der makroökonomischen Korrekturverfahren, soweit diese sanktionsbewehrt sind, ausgenommen werden. Vom Anwendungsbereich des europäischen Wettbewerbsrechts sind zudem die Sektoren der öffentlichen Daseinsvorsorge auszunehmen.

\section{Das Demokratiedefizit geht tief}

Viel zu sagen wäre über die Behandlung des europäischen Demokratiedefizits. In der derzeitigen Fassung benennt der Entwurf das Defizit korrekt, will es aber durch (1) mehr Rechte des Europäischen Parlaments, (2) europäische Referenden und (3) Bekämpfung des Lobbyings gegenüber der europäischen Gesetzgebung beheben. Das europäische Demokratiedefizit geht aber wesentlich tiefer. Es wurzelt in der spezifischen „Überkonstitutionalisierung” (Dieter Grimm in diesem Band) der EU, also darin, dass ein Großteil europäischer Entscheidungen das politische System der EU gar nicht erst erreicht, sondern als Verfassungsvollzug vom EuGH angeordnet wird oder sich durch Maßnahmen anderer demokratisch nicht legitimierter Organe wie der EZB vollzieht. Hier helfen auch mehr EPRechte in der europäischen Gesetzgebung nicht.

Betrachten wir folgenden Auszug eingehender (Zeilen 1733-1737):

„Die EU kann durch Bewegung von unten für eine unteilbare Demokratie und soziale Gerechtigkeit verändert werden. Bereits früher konnten durch den Druck von Gewerkschaften und sozialen Bewegungen neoliberale Vorstöße zur Privatisierung der Wasserversorgung (2014) und die sogenannte Bolkestein-Richtlinie (2006) zur Liberalisierung von Dienstleistungen im EU-Binnenmarkt verhindert bzw. abgeschwächt werden."

Leider zeigt das Beispiel der Dienstleistungsrichtlinie genau das nicht - sondern vielmehr, wie hartnäckig abgeschottet das EU-Binnenmarktrecht vor demokratischer Einflussnahme ist. Der ursprüngliche Entwurf der Dienstleistungsrichtlinie kodifizierte - das ist das typische Muster der Gesetzgebung zum Binnenmarkt - vor allem vorangegangene EuGH-Rechtsprechung. Gewerkschaften, Bewegungen und wackere EP-Parlamentarier kämpften die explizite Nennung des Herkunftslandprinzips aus der Richtlinie heraus und waren stolz, diese Auseinandersetzung - scheinbar - gewonnen zu haben. Der EuGH hat diesen Umstand aber schlicht ignoriert und blieb bei seiner Rechtsprechung, faktisch wurde nichts abgeschwächt oder verhindert.

Diese Einsicht soll uns nun nicht dazu verleiten, die Flinte ins Korn zu werfen. Sie zeigt aber, was für 
dicke Bretter zu bohren sind, sobald in der EU die Binnenmarktregeln ins Spiel kommen. Auch deshalb warnen wir vor dem Ruf nach einer "Verfassung" und raten dringend zu einer eigenständigen Befassung mit den Binnenmarktfreiheiten. Zudem ist notwendig, das EU-Demokratiedefizit in seiner Tiefe erkennen und ehrlich benennen.

Und es bleibt das grundlegende Problem, dass wesentliche gesellschaftliche Voraussetzungen für Demokratie auf EU-Ebene nicht gegeben sind: eine gemeinsame politische Öffentlichkeit und Zivilgesellschaft auf Basis einer gemeinsamen Sprache, hinreichende Gleichartigkeit der wirtschaftlichen, sozialen, rechtlichen und politischen Bedingungen und Traditionen, letztlich die Bereitschaft der nationalen Gesellschaften und Mitgliedstaaten, die übergeordnete Geltung von EU-Entscheidungen zu akzeptieren. EU-Vereinheitlichung trotzdem immer weiter voranzutreiben, läuft Gefahr, Demokratie aushöhlen statt zu stärken.

Ein Schlüssel zur Verteidigung demokratischer Prozeduren liegt unserer Überzeugung nach daher darin, das Subsidiaritätsprinzip auch und gerade von links ernst zu nehmen. Neben dem Plädoyer für die Maximierung des sozialen Outputs des europäischen Mehrebenensystems haben wir damit ein zweites Kriterium an der Hand, mit dem entschieden werden sollte, wo die Forderung nach Verlagerung von Kompetenzen und Befugnissen an die EU aus progressiver Sicht sinnvoll erscheint und wo nicht. Die europäischen Organe sollten zum Handeln ermächtigt werden, wo es genuin transnationale Probleme zu lösen gilt; und wo es progressiven ökonomischen, sozialen, ökologischen und friedenspolitischen Zwecken dient. Wo das nicht der Fall ist, sollte von der Forderung nach weiteren Verlagerungen Abstand genommen werden. Und wo die entsprechenden Befugnisse bereits bei der EU liegen, darf auch der Ruf nach Rückverlagerungen („selektiver Rückbau“) kein Tabu sein. Das gilt namentlich auch für die Aufrüstungsbestrebungen im Rahmen der PESCO (ständige strukturierte Zusammenarbeit in der Sicherheits- und Verteidigungspolitik).

\section{Ein soziales Kernprojekt für die EU}

Wir schlagen vor, ein soziales „Leuchtturmprojekt" für die EU zu identifizieren und es in den kommenden Jahren offensiv zu bewerben. Die Verständigung auf ein solches Projekt könnte dazu beitragen, die progressiven Parteien, Organisationen und Bewegungen in der Europafrage enger zusammenzuführen als in der Vergangenheit. Das schwerwiegendste soziale Defizit in der EU (nicht: der EU, die europäischen Organe haben das Defizit nicht verursacht) besteht in der hohen Anzahl von (fast immer: ärmeren) EU-Ländern ohne sozialen Mindestschutz. Die EU sollte erhebliche Mittel in den Strukturfonds freimachen, sie zusätzlich aufstocken (statt sie, wie in der derzeitigen Finanzplanung vorgesehen, zu kürzen) und mit diesen den Aufbau sozialer Grundsicherungen in den ärmeren EU-Ländern unterstützen.

Aus unserer Sicht ist dieses Projekt in vielerlei Hinsicht gut aufgestellt:

- Es setzt am offensichtlichsten sozialen Missstand der EU an.

- Es verknüpft die Definition von Mindeststandards mit transnationalen Hilfen. Derzeit laufen die meisten im Entwurf genannten Programmpunkte lediglich darauf hinaus, die Mitgliedstaaten zu etwas zu verpflichten. Das Projekt will helfen statt bestrafen und dezentrale Autonomie 
bewahren statt von oben vereinheitlichen.

- Bei dem Projekt wäre sichergestellt, dass die Transfers von reich zu arm verlaufen würden. Bei der diskutierten Europäischen Arbeitslosenversicherung, zu der sich das Programm in der derzeitigen Fassung bekennt (Zeilen 461-467), wäre das keineswegs so. In vielerlei Hinsicht ist das vorgeschlagene Projekt zielgenauer, wenn es darum geht, Transfers für soziale Zwecke einzusetzen, und zudem deutlich besser kommunizierbar.

- Das Projekt sollte über unterschiedliche Ländergruppen hinweg unter den Linken konsensfähig, aber auch über diese hinaus anschlussfähig sein. Auch in den reicheren Mitgliedstaaten sollten wir Mitstreiter für europäische Hilfen beim Aufbau des sozialen Mindestschutzes gewinnen können. Denn mit den Westbalkanländern stehen EU-Beitrittskandidaten vor der Tür, die noch einmal deutlich ärmer sind als Bulgarien und Rumänien. Wer Armutsmigration begrenzen will, wird daran mithelfen müssen, dass die Ärmsten in den ärmsten europäischen Ländern nicht vor dem Nichts stehen - anders geht es nicht.

- Die Bilanz der bisherigen EU-Strukturpolitik ist gemischt. Dieser Umstand droht zu einem Einfallstor für die Kürzung transnationaler Hilfen zu werden. Das Projekt weist der Strukturpolitik einen für alle einsehbaren, genuin sozialen Zweck zu.

\section{Den Konflikt über die Migrationspolitik pazifizieren}

Wir schließen mit einer Anmerkung zu einem Seitenaspekt, der den Bonner Parteitag gewiss beschäftigen wird. Die auseinanderstrebenden Haltungen in der Migrationsfrage haben die Partei DIE LINKE seit 2015 an den Rand der Spaltung geführt. Das vergiftete Klima in der Linkspartei hatte destruktive Wirkungen weit über die Partei hinaus. Wir hoffen, dass es gelingt, den Disput über diese Detailfrage in geordnete Bahnen zu lenken. Der in diesem Kommentar diskutierte Entwurf zeigt bei der Thematisierung des bedingungslosen Grundeinkommens (Zeilen 480-484), dass es möglich ist, umstrittene Themen als solche zu kennzeichnen. Weder die Partei DIE LINKE noch andere progressive Organisationen müssen sich dafür schämen, so etwas offen zu bekennen.

Leider sind Zweifel angebracht, ob der vom Parteivorstand der Linkspartei vorgelegte Entwurf geeignet ist, den grotesken Streit über die Zuwanderungsfrage zu pazifizieren. Denn anders als im Leitantrag zum Leipziger Parteitag vom Juni 2018 erfolgt die Forderung nach „offenen Grenzen für alle Menschen" im vorliegenden Text nunmehr ohne Bezug zur Fluchtmigration (Z 1579-80). Wir hoffen, dass sich in dieser Frage Vernunft Bahn bricht und kluge Formulierungen gefunden werden, mit denen alle Beteiligten gut leben können.

Wir danken Klaus Dräger für wertvolle Hinweise.

[1] In diesen Entscheidungen aus dem Jahr 2007 hatte der Europäische Gerichtshof die Ausübung des Streikrechts dem für Binnenmarktstörungen entwickelten Rechtfertigungstest unterworfen. Arbeitskämpfe können demnach illegal sein, wenn sie die Dienstleistungsfreiheit (Laval) oder die Niederlassungsfreiheit (Viking) unverhältnismäßig stören. 
Veröffentlicht am: 16.01.2019

Erschienen unter: https://makroskop.eu/2019/01/welches-europa-wollen-wir/ 